\title{
A-Stability of a Class of Methods for the Numerical Integration of Certain Linear Systems of Ordinary Differential Equations
}

\author{
By M. R. Crisci and E. Russo
}

\begin{abstract}
This paper is concerned with the analysis of the stability of a class of one-step integration methods, originated by the Lanczos tau method and applicable to particular linear differential systems.

It is proved that these methods are $A$-stable for every order.
\end{abstract}

1. Introduction. In [1] the authors derived from the Lanczos tau method a class of one-step methods for the numerical integration of linear differential systems with polynomial coefficients.

This paper is concerned with the stability analysis of the above methods.

As it is known, the stability properties of a method are determined through its application to the test equation $y^{\prime}-\lambda y=0$ [3]. In fact, let us consider the general differential system $y^{\prime}-f(x, y)=0$, and let $g(x)$ be the particular solution we attempt to compute. The local behaviour of this system is determined, to a first approximation, by the solution of the variational equation $y^{\prime}-J(x) y=0$, where $J(x)=f_{y}(x, g(x))$ is the Jacobian matrix. If $J(x)$ varies slowly, locally it can be regarded as a constant matrix $A$, and the equation is modelled by $y^{\prime}-A y=0$. Let us further assume that $A$ is diagonalizable and let $P$ be the similarity transformation which takes $A$ to the diagonal form $\Lambda$. Then the substitution $u=P y$ changes the above system to $u^{\prime}-\Lambda u=0$, such that each equation is independent of the others and it is exactly of the form of the test equation.

The region of absolute stability of the method is the set of $h \lambda$ such that the obtained numerical solution is decaying.

In this paper, in order to analyze the stability, we will give in Section 2 a simplified illustration of the class of methods developed in [1] and applied to a single equation. However, it is not prejudicial to the outcome.

In Section 3 it is proved that the stability region contains the whole of the left-hand half-plane $\operatorname{Re}(h \lambda)<0$, and therefore the methods are $A$-stable for every order.

2. The Class of Methods. Let us review briefly the method for a single first-order differential equation; for the sake of clarity we restrict ourselves to the results essential to follow the stability analysis. The complete treatment of the method can be found in [1].

Received May 27, 1980; revised February 10, 1981 and July 29, 1981.

1980 Mathematics Subject Classification. Primary 65L05. 
Let $D$ be a first-order linear differential operator which maps polynomials into polynomials, and let us consider the following initial value problem:

$$
\left\{\begin{array}{l}
D y(x)=0, \quad x_{0} \leqslant x \leqslant b, \\
y\left(x_{0}\right)=y_{0} .
\end{array}\right.
$$

Let us subdivide the interval $\left[x_{0}, b\right]$ by a finite set of distinct points $x_{n+1}=x_{n}+h$, and let $y_{n}$ be the approximate value in $x_{n}$ of the solution.

Let us consider, for each subinterval $\left[x_{n}, x_{n+1}\right]$, the perturbed problem:

$$
\left\{\begin{array}{l}
D y_{n}^{m}(x)=\tau_{n} \tilde{T}_{m}(x), \quad x_{n} \leqslant x \leqslant x_{n+1}, \\
y_{n}^{m}\left(x_{n}\right)=y_{n},
\end{array}\right.
$$

where $\tilde{T}_{m}(x)$ is the Chebyshev polynomial defined in $\left[x_{n}, x_{n+1}\right], \tau_{n}$ is a parameter to be determined, and $y_{n}^{m}(x)$ is a polynomial of degree $m$, which satisfies exactly the perturbed problem (2.2) (2.2'). The method determines $y_{n+1}$ as the value in $x_{n+1}$ of $y_{n}^{m}(x)$. It is of order $m$ in the sense that if the exact solution of (2.1) is itself a polynomial of degree $m$, the method will reproduce it.

Let us introduce the canonical polynomials $Q_{k}(x)$, defined by the relation*

$$
D Q_{k}(x)=x^{k}, \quad k \in N_{0} \text {. }
$$

These polynomials, which can be easily generated by means of a recurrence relation [1], are independent of the integration interval $\left[x_{n}, x_{n+1}\right]$, and allow a straight construction of $y_{n}^{m}(x)$.

In fact, because of the linearity of $D$, the solution of (2.2) can be expressed through

$$
y_{n}^{m}(x)=\tau_{n}\left(\tilde{c}_{0}^{m} Q_{0}(x)+\cdots+\tilde{c}_{m}^{m} Q_{m}(x)\right),
$$

where $\tilde{c}_{k}^{m}$ is the coefficient of $x^{k}$ in $\tilde{T}_{m}(x)$.

The parameter $\tau_{n}$ is calculated by imposing that $y_{n}^{m}(x)$ satisfies the initial condition $\left(2.2^{\prime}\right)$, and so is given by

$$
\tau_{n}=\frac{y_{n}}{\sum_{k=0}^{m} \tilde{c}_{k}^{m} Q_{k}\left(x_{n}\right)} .
$$

Therefore, for (2.4), (2.5), $y_{n+1}$ is given by

$$
y_{n+1}=y_{n} \frac{\sum_{k=0}^{m} \tilde{c}_{k}^{m} Q_{k}\left(x_{n+1}\right)}{\sum_{k=0}^{m} \tilde{c}_{k}^{m} Q_{k}\left(x_{n}\right)}
$$

and depends on $h$ through the coefficients $\tilde{c}_{k}^{m}$.

(2.6) is, then, the formula of order $m$ of the class under consideration.

3. $A$-Stability of the Method. Let us apply the method (2.6) of order $m$, with $m$ arbitrarily fixed, to the test equation

$$
y^{\prime}-\lambda y=0
$$

\footnotetext{
*This is a simplified definition, sufficient for our purposes. In general, some $Q_{k}(x)$ can remain undefined for a finite set $S$ of index $k$, and a linear combination of powers of $x$ with exponents in $S$ is to be added at the left hand of (2.3). For further details cf. [1] and [5].
} 
First of all, an expression for the canonical polynomials is required. For the particularity of (3.1), $Q_{k}(x)$ can be easily derived from (2.3) and are given by

$$
Q_{k}(x)=-\sum_{j=0}^{k} \frac{k !}{j ! \lambda^{k-j+1}} x^{j}, \quad k \in N_{0} .
$$

From (2.6) and (3.2), with some algebraic manipulations, and putting

$$
\tilde{T}_{m}^{(k)}(x)=\frac{d^{k}}{d x^{k}} \tilde{T}_{m}(x)
$$

it follows that

$$
y_{n+1}=y_{n} \frac{\sum_{k=0}^{m} \frac{1}{\lambda^{k}} \tilde{T}_{m}^{(k)}\left(x_{n+1}\right)}{\sum_{k=0}^{m} \frac{1}{\lambda^{k}} \tilde{T}_{m}^{(k)}\left(x_{n}\right)} .
$$

Denoting by $T_{m}^{*}(x)$ the Chebyshev polynomial defined in $[0,1]$, it is obvious that

$$
\tilde{T}_{m}^{(k)}(x)=\frac{1}{h^{k}} T_{m}^{*(k)}\left(\frac{1}{h}\left(x-x_{n}\right)\right),
$$

and so, putting $q=(h \lambda)^{-1},(3.3)$ can be written

$$
y_{n+1}=y_{n} \frac{\sum_{k=0}^{m} T_{m}^{*(k)}(1) q^{k}}{\sum_{k=0}^{m} T_{m}^{*(k)}(0) q^{k}} .
$$

Let $F_{m}(q)$ be defined by

$$
F_{m}(q)=\frac{\sum_{k=0}^{m} T_{m}^{*(k)}(1) q^{k}}{\sum_{k=0}^{m} T_{m}^{*(k)}(0) q^{k}} .
$$

The denominator of $F_{m}(q)$ can be written as

$$
G_{m}(q)=\sum_{k=0}^{m} k ! c_{k}^{m} q^{k},
$$

where $c_{k}^{m}$ is the coefficient of $x^{k}$ in $T_{m}^{*}(x)$.

Let us denote, as usual, the current variable of the polynomial $G_{m}$ by $z$. The following lemma, which will be useful hereafter, can be proved.

LEMMA 1. The polynomial $G_{m}(z)$ has all the zeros in the half-plane $\operatorname{Re}(z)>0$.

Proof. The polynomial

$$
\pi(z)=\sum_{k=0}^{m} k !\left|c_{k}^{m}\right| z^{k}
$$

has its zero opposite to those of $G_{m}(z)$, because of the property of $c_{k}^{m}$ to alternate signs. So it will be equivalent to prove that $\pi(z)$ has all its zeros in the half-plane $\operatorname{Re}(z)<0$, that is, it is a stable polynomial.

Let $\beta_{1}, \beta_{2} \cdots \beta_{m}$ be the zeros of the polynomial

$$
\Gamma(z)=\sum_{k=0}^{m}\left|c_{k}^{m}\right| z^{k} .
$$

Since $\Gamma(z)=(-1)^{m} T_{m}^{*}(-z), \beta_{i}$ are all real, simple and lying in $]-1,0[$. 
The polynomial

$$
\Theta(z)=\sum_{k=0}^{m} \frac{1}{(m-k) !} z^{k}
$$

is a stable polynomial, as can be readily verified by the Routh-Hurwitz criterion.

The coefficients of $\pi(z)$ can be expressed combining those of $\Gamma(z)$ and $\Theta(z)$. In fact, if we put

$$
\gamma_{k}=c_{k}^{m} /\left(\begin{array}{c}
m \\
k
\end{array}\right), \quad \Theta_{k}=k !
$$

then

$$
\begin{aligned}
& \Gamma(z)=\sum_{k=0}^{m}\left(\begin{array}{c}
m \\
k
\end{array}\right) \gamma_{k} z^{k}, \\
& \Theta(z)=\frac{1}{m !} \sum_{k=0}^{m}\left(\begin{array}{c}
m \\
k
\end{array}\right) \Theta_{k} z^{k}, \\
& \pi(z)=\sum_{k=0}^{m}\left(\begin{array}{c}
m \\
k
\end{array}\right) \gamma_{k} \Theta_{k} z^{k}
\end{aligned}
$$

Now, applying a known theorem of Szegö [4], every zero $\delta_{i}$ of $\pi(z)$ has the form $\delta_{i}=-\alpha \beta_{i}$, where $\alpha$ is a suitably chosen point of the half-plane of the zeros of $\Theta(z)$, that is, the half-plane $\operatorname{Re}(z)<0$, and $\beta_{i}$ is a negative real number, since it is a zero of $\Gamma(z)$.

Therefore $\pi(z)$ is a stable polynomial, and the result holds.

That being stated, the main result can be proved.

THEOREM 1. The methods are A-stable for every order $m$.

Proof. As is known, the method is $A$-stable if and only if $\left|F_{m}(q)\right| \leqslant 1$ for $\operatorname{Re}(q) \leqslant 0$. This in turn follows if and only if the following two conditions hold:

(i) $\left|F_{m}(q)\right| \leqslant 1$ on $\operatorname{Re}(q)=0$,

(ii) $F_{m}(q)$ is analytic for $\operatorname{Re}(q)<0$,

because the maximum modulus theorem can be applied to establish boundedness.

In order to prove (i) some properties of $T_{m}^{*(k)}(x)$ will be needed. In particular it is easily verified that

$$
\begin{gathered}
\left|T_{m}^{*(k)}(0)\right|=\left|T_{m}^{*(k)}(1)\right|, \\
\operatorname{sgn}\left(T_{m}^{*(k)}(0)\right)=(-1)^{m+k}, \\
\operatorname{sgn}\left(T_{m}^{*(k)}(1)\right)=1 .
\end{gathered}
$$

Therefore (3.6) can be written:

$$
F_{m}(q)=\frac{\sum_{k=0}^{m} T_{m}^{*(k)}(1) q^{k}}{(-1)^{m} \sum_{k=0}^{m}(-1)^{k} T_{m}^{*(k)}(1) q^{k}}
$$

For $\operatorname{Re}(q)=0, F_{m}(q)$ is the ratio of two conjugate complex numbers, and then (i) holds. (ii) is also satisfied, as has been proved by Lemma 1.

The theorem holds. 
Istituto di Matematica

Università di Napoli

Via Mezzocannone, 8

80134 Napoli, Italy

1. M. R. CRISCI \& E. RUSSO, “A class of methods for the numerical integration of certain linear systems of ordinary differential equations." (To appear.)

2. C. Lanczos, "Trigonometric interpolation of empirical and analytical functions," J. Math. Phys., v. 17, 1938, pp. 123-199.

3. T. LAPIDUS \& J. H. Seinfeld, "Numerical solution of ordinary differential equations," Mathematics in Science and Engineering, vol. 75, Academic Press, New York, 1971.

4. M. MARden, The Geometry of the Zeros of a Polynomial in a Complex Variable, Math. Surveys, no. 3, Amer. Math. Soc., Providence, R. I., 1949.

5. E. ORTIZ, "The tau method," SIAM J. Numer. Anal., v. 6, 1969, pp. 480-492.

6. E. ORTIZ, "Canonical polynomials in the Lanczos tau method," Studies in Numerical Analysis, Academic Press, London, 1974.

7. K. WRIGHT, "Some relationships between implicit Runge-Kutta, collocation and Lanczos $\tau$ methods, and their stability properties," BIT, v. 10, 1970, pp. 217-227. 